\title{
Primer estudio de la riqueza de coleópteros en un bosque de Polylepis tomentella del distrito de Chaviña (Ayacucho, Perú)
}

\author{
Cristhian Rossi'; Iván Galindo'; Gabriela Huaman'; Blanca Cuadros'; Yuriko \\ ORTEGA $^{1, \otimes}$; Eliana QuisPitupaC ${ }^{12}$ \& Norberta MarTíneZ ${ }^{12}$ \\ ${ }^{1}$ Laboratorio de Entomología, Departamento de Zoología, Facultad de Ciencias Biológicas, Universidad Nacional Mayor de \\ San Marcos. Lima, Perú. ${ }^{2}$ Instituto de Investigación de Ciencias Biológicas Antonio Raimondi (ICBAR), Facultad de Ciencias \\ Biológicas, Universidad Nacional Mayor de San Marcos. Lima, Perú.
}

\begin{abstract}
Resumen. Los bosques de Polylepis son ecosistemas vulnerables que están bajo una presión antropogénica fuerte. Se encuentran fragmentados y forman parches en laderas de montañas y roquedales altoandinos. Allí se sitúan los bosques de Polylepis tomentella, distribuidos en los departamentos de Ayacucho, Apurímac y Arequipa. En el año 2010, se evaluó la estructura comunitaria del orden Coleoptera en un parche representativo del distrito de Chaviña. Se realizaron dos evaluaciones (época húmeda y seca) en las que se establecieron diez transectos de muestreo y se emplearon trampas de caída, trampas con cebo, trampas cromotrópicas, colecta activa y trampas Malaise. Se identificaron 45 especies distribuidas en 18 familias, de las cuales Curculionidae y Carabidae fueron las más representativas. La riqueza estimada osciló entre 43 y 47 especies. El mejor estimador de la riqueza fue ACE. Los datos se pueden usar para estudios posteriores de diversidad y también para estimar el grado de perturbación de estos bosques relictos.
\end{abstract}

[Palabras clave: escarabajos, queñoa, riqueza, frecuencia, curvas de acumulación]

\begin{abstract}
First study of the coleopterans richness of Polylepis tomentella from Chaviña district (Ayacucho, Perú). Polylepis woodlands are vulnerable ecosystems that are under strong anthropogenic pressure. These woodlands are fragmented, forming patches on the slopes of high andean and rocky mountains, where we find Polylepis tomentella. They are distributed in the departments of Ayacucho, Apurimac and Arequipa. In 2010, we evaluated the community structure of the order Coleoptera in a representative patch of the Chaviña district. Two surveys (wet and dry season) were carried out. Ten sampling stations were established and pitfall traps, bait traps, chromotropic traps, active collection and Malaise traps were used. Forty-five species were identified and distributed in 18 families, being Curculionidae and Carabidae the most representative. Estimated richness was between 43 and 47 species. The best richness estimator was ACE. These data can be useful for subsequent diversity studies and also to estimate the degree of disturbance of these woodlands.
\end{abstract}

[Keywords: beetles, queñoa, richness, frequency, accumulation curves]

\section{INTRODUCCIÓN}

Los bosques de Polylepis constituyen ecosistemas únicos, de distribución restringida a lo largo de los Andes. Abarcan desde Venezuela hasta el norte de Chile y el centro de la Argentina. Conforman enclaves forestales de gran importancia florística y ecológica (Barrera 2001). Ocurren a grandes altitudes, superando los $5000 \mathrm{~m} \mathrm{~s}$. n. m. y se distribuyen en parches de vegetación leñosa rodeados por comunidades de páramos, pastos y arbustos (Ellenberg 1958; Stotz et al. 1996; Velez et al. 1998; Kessler 2002) que en el pasado pudieron haber abarcado áreas más extensas (Perrault 1994).

En el Perú, los bosques de Polylepis presentan una amplia distribución en la zona norte y sur de los Andes (Bustamante et al. 2007; Bustamante et al. 2017). Entre ellos, los de Polylepis tomentella Wedd., ubicados en los departamentos de Ayacucho, Apurímac y Arequipa (Mendoza and Cano 2011; Zutta et al. 2012), forman fragmentos aislados que reciben un considerable impacto causado por la tala excesiva y otras actividades que se realizan en zonas cercanas a su área de distribución (Mendoza and Roque 2007). Para un adecuado manejo de estos bosques es necesario conocer su estado de conservación, que puede estar basado en indicadores biológicos (e.g., la riqueza de la entomofauna). Los insectos son el grupo más representativo entre los animales y ocupan diversos nichos ecológicos, en los que realizan procesos naturales importantes para la dinámica de los ecosistemas (Triplehorn et al. 2005; Gullan and Cranston 2010).

Editora asociada: Erica Cuyckens 
Dentro de la Clase Insecta, los coleópteros son el grupo más abundante y diverso. Para el Perú se reportan 99 de las 165 familias descritas (Lawrence and Newton 1995; Chaboo 2015). Los coleópteros son empleados en estudios de biología de la conservación (Didham et al. 1998; Lozada et al. 2004) porque presentan una elevada abundancia y diversidad en todo tipo de ecosistema, por ser sensibles a las perturbaciones ambientales y presentar ciclos de vida cortos (Méndez et al. 2012), por ser colectados fácilmente con metodologías estandarizadas, por conocerse su taxonomía (Hamel et al. 2009) y por cumplir roles específicos en el hábitat (e.g., ser depredadores y presas de diversos organismos) (CBG 2008; Meneses and Camacho 2016; Qodri et al. 2016). Este orden es considerado como un indicador ecológico clave, y las familias Carabidae y Scarabaeidae son aquellas en las que, hasta hoy, se centró la mayor parte de las investigaciones (Fuentes and Camero 2006; Nichols et al. 2007; Otavo et al. 2013; Mora 2015). Es posible que los bosques de $P$. tomentella alberguen una riqueza considerable de escarabajos. Dada la importancia de este orden, el presente estudio tiene por objetivo dar a conocer la riqueza de coleópteros de este bosque fragmentado ubicado en el departamento de Ayacucho.

\section{Materiales y MÉTOdos}

\section{Área de estudio}

Este trabajo se realizó en el distrito de Chaviña, en la provincia de Lucanas, departamento de Ayacucho, en un área representativa del bosque de $P$. tomentella. Esta zona se ubicó en $14^{\circ} 54^{\prime} 27^{\prime \prime}$ ' S y $73^{\circ} 53^{\prime} 57^{\prime \prime}$ O, entre los 4000 y 4200 m s. n. m. El área se localiza en la provincia biogeográfica de Puna Subtropical (Zamora 1996), que posee un clima de tundra seca de alta montaña.

\section{Metodología}

En el año 2010 se realizaron dos evaluaciones: una en abril (época húmeda) y otra en septiembre (época seca). Se establecieron 10 transectos de $100 \mathrm{~m}$ de longitud. En cada uno se instalaron siete trampas de caída (sin cebo), dos necrotrampas con cebo de pescado, una coprotrampa con excremento humano y dos trampas cromotrópicas amarillas. Estas trampas estuvieron activas por 72 horas. Además, en el área de estudio se realizó una colecta activa (captura manual y con malla entomológica) por 30 minutos en cada transecta y se instalaron dos trampas Malaise (colecta de insectos por intercepción de vuelo). Estas trampas permanecieron activas por 72 horas. Las muestras se depositaron en el Laboratorio de Entomología de la Facultad de Ciencias Biológicas de la Universidad Nacional Mayor de San Marcos.

\section{Estimación de la riqueza de especies}

Los individuos fueron identificados hasta el nivel de especie cuando esto era posible; si no, sólo hasta el nivel taxonómico mejor conocido. La riqueza fue estimada mediante el desarrollo de curvas de acumulación de especies. Para este análisis se tomaron en cuenta los datos colectados con trampas pasivas (se excluyó la colecta activa y las trampas Malaise) por representar un esfuerzo de muestreo equitativo para las dos épocas. Se escogieron los estimadores no paramétricos Chao 2, ACE e ICE porque presentan un desempeño mejor que otras funciones asintóticas al estimar la riqueza de especies en un área pequeña muestreada exhaustivamente (López and Williams 2006; Gonzáles et al. 2010; Gotelli and Colwell 2011). Los datos fueron analizados en el programa EstimateS vers. 9.0 (Colwell 2013). Los resultados fueron exportados al programa Microsoft ${ }^{\circledR}$ Office Excel para graficar las curvas y realizar las interpretaciones correspondientes.

\section{Resultados y DiscusióN}

En Sudamérica, algunos estudios se enfocaron en evaluar la fauna acompañante de bosques de Polylepis (Ashe and Leschen 1995; Barrera 2001; Lloyd 2008) con la finalidad de estimar su grado de conservación y brindar información sobre las posibles causas que afectan el equilibrio de estos ecosistemas fragmentados (Perrault 1994; Velez et al. 1998; Meneses and Camacho 2016). A menudo, los estudios entomológicos se orientan a las familias Scarabaeidae, Staphylinidae y Carabidae (Ashe and Leschen 1995), como los realizados en bosques de Polylepis sericea Wedd. (Barrera 2001) y Polylepis quadrijuga Bitter (Meneses and Camacho 2016) en Venezuela y Colombia, respectivamente.

En el Perú existe un escaso conocimiento de la entomofauna en los bosques de Polylepis, salvo por algunos inventarios realizados en los departamentos de Ancash, Ayacucho y Puno. Estos inventarios están orientados a 
Polylepis canoi W. Mendoza, Polylepis incarum (Bitter) M. Kessler and Schmidt-Leb., Polylepis pepei B. Simpson, Polylepis sericea, Polylepis triacontandra Bitter, Polylepis weberbaueri Pilger y Polylepis sp. (ECOAN 2005, 2006, 2007; Bustamante et al. 2017), por lo que este trabajo representa la primera incursión en evaluar la estructura comunitaria del orden
Coleoptera en el bosque de Polylepis tomentella del departamento de Ayacucho.

Se registraron 45 especies (11 identificadas hasta el nivel de especie), agrupadas en 18 familias (Tabla 1), sin considerar a los Staphylinidae por ser parte de un trabajo de tesis que se encuentra en desarrollo. En los

Tabla 1. Lista de coleópteros identificados del bosque de Polylepis tomentella durante las dos épocas de muestreo. n: abundancia relativa; Prom: promedio de individuos; DE: desviación estándar; FR\%: frecuencia relativa.

Table 1. List of identified coleopterans from Polylepis tomentella woodland during the two sampling periods. n: relative abundance; Prom: individuals average; DE: standard deviation; FR\%: relative frequency.

\begin{tabular}{|c|c|c|c|c|c|c|c|}
\hline \multirow[b]{2}{*}{ Familia } & \multirow[b]{2}{*}{ Especie } & \multicolumn{3}{|c|}{ Húmeda } & \multicolumn{3}{|c|}{ Seca } \\
\hline & & $\mathrm{n}$ & Prom $\pm \mathrm{DE}$ & $\mathrm{FR} \%$ & $\mathrm{n}$ & Prom \pm DE & $\mathrm{FR} \%$ \\
\hline ANTHICIDAE & Anthicinae sp. & 1 & $0.07 \pm 0.27$ & 7.14 & 0 & & \\
\hline BRENTIDAE & Apioninae sp. & 1 & $0.07 \pm 0.27$ & 7.14 & 1 & $0.07 \pm 0.27$ & 7.14 \\
\hline \multirow[t]{4}{*}{ CARABIDAE } & Bembidion sp.1 & 12 & $0.86 \pm 1.51$ & 35.71 & 4 & $0.29 \pm 0.47$ & 28.57 \\
\hline & Bembidion sp.2 & 11 & $0.79 \pm 2.39$ & 21.43 & 0 & & \\
\hline & Bembidion sp.3 & 7 & $0.50 \pm 0.76$ & 35.71 & 0 & & \\
\hline & Lebiini sp. & 0 & & & 9 & $0.64 \pm 1.08$ & 28.57 \\
\hline \multirow[t]{5}{*}{ CHRYSOMELIDAE } & Pachybrachis sp. & 6 & $0.43 \pm 0.76$ & 28.57 & 0 & & \\
\hline & Chaetocnema sp. & 1 & $0.07 \pm 0.27$ & 7.14 & 0 & & \\
\hline & Epitrix sp. & 5 & $0.36 \pm 1.08$ & 14.29 & 0 & & \\
\hline & Longitarsus sp. & 14 & $1.00 \pm 2.54$ & 21.43 & 0 & & \\
\hline & Phyllotreta sp. & 13 & $0.93 \pm 3.47$ & 7.14 & 0 & & \\
\hline \multirow[t]{5}{*}{ COCCINELLIDAE } & Cycloneda sp. & 2 & $0.14 \pm 0.36$ & 14.29 & 0 & & \\
\hline & Cycloneda arcula (Erichson, 1847) & 12 & $0.86 \pm 2.66$ & 21.43 & 2 & $0.14 \pm 0.36$ & 14.29 \\
\hline & Eriopis nobilis Mader, 1958 & 0 & & & 1 & $0.07 \pm 0.27$ & 7.14 \\
\hline & Eriopis sp. & 5 & $0.36 \pm 0.50$ & 35.71 & 2 & $0.14 \pm 0.36$ & 14.29 \\
\hline & Hippodamia convergens (Guerin-Meneville, 1842) & 3 & $0.21 \pm 0.58$ & 14.29 & 1 & $0.07 \pm 0.27$ & 7.14 \\
\hline \multirow[t]{9}{*}{ CURCULIONIDAE } & Listroderini sp. & 1 & $0.07 \pm 0.27$ & 7.14 & 0 & & \\
\hline & Puranius sp. & 2 & $0.14 \pm 0.36$ & 14.29 & 0 & & \\
\hline & Cylydrorhinini sp. & 1 & $0.07 \pm 0.27$ & 7.14 & 0 & & \\
\hline & Amitrus jelskei (Kirsch, 1889) & 3 & $0.21 \pm 0.58$ & 14.29 & 0 & & \\
\hline & Amphideritus sp. & 2 & $0.14 \pm 0.36$ & 14.29 & 0 & & \\
\hline & Asymmathetes sp. & 95 & $6.79 \pm 16.22$ & 57.14 & 0 & & \\
\hline & Piazomiina sp. & 3 & $0.21 \pm 0.80$ & 7.14 & 0 & & \\
\hline & Listroderini sp. & 1 & $0.07 \pm 0.27$ & 7.14 & 0 & & \\
\hline & Scolytinae sp. & 2 & $0.14 \pm 0.53$ & 7.14 & 1 & $0.07 \pm 0.27$ & 7.14 \\
\hline CYBOCEPHALIDAE & Cybocephalidae sp. & 22 & $1.57 \pm 4.24$ & 35.71 & 0 & & \\
\hline HISTERIDAE & Euspilotus cf. decoratus Erichson, 1834 & 78 & $5.57 \pm 12.15$ & 28.57 & 4 & $0.29 \pm 0.73$ & 14.29 \\
\hline \multirow[t]{2}{*}{ HYDRAENIDAE } & Gymnochthebius sp. & 1 & $0.07 \pm 0.27$ & 7.14 & 0 & & \\
\hline & Ochthebius sp. & 1 & $0.07 \pm 0.27$ & 7.14 & 0 & & \\
\hline HYDROPHILIDAE & Sphaeridiinae sp. & 1 & $0.07 \pm 0.27$ & 7.14 & 0 & & \\
\hline LATRIDIIDAE & Latridiidae sp. & 1 & $0.07 \pm 0.27$ & 7.14 & 0 & & \\
\hline LEIODIDAE & Leiodini sp. & 2 & $0.14 \pm 0.36$ & 14.29 & 0 & & \\
\hline \multirow[t]{2}{*}{ MELYRIDAE } & Listrus sp. & 16 & $1.14 \pm 2.35$ & 21.43 & 0 & & \\
\hline & Astylus subannulatus Pic, 1929 & 18 & $1.29 \pm 3.81$ & 14.29 & 0 & & \\
\hline NITIDULIDAE & Nitidulinae sp. & 0 & & & 1 & $0.07 \pm 0.27$ & 7.14 \\
\hline \multirow[t]{3}{*}{ SCARABAEIDAE } & Podotenus sp. & 51 & $3.64 \pm 7.55$ & 35.71 & 5 & $0.36 \pm 1.34$ & 7.14 \\
\hline & Canthon sp. & 5 & $0.36 \pm 1.08$ & 14.29 & 0 & & \\
\hline & Scybalophagus rugosus (Blanchard, 1845) & 6 & $0.43 \pm 0.85$ & 21.43 & 0 & & \\
\hline SILPHIDAE & Oxelytrum anticola (Guerin-Meneville, 1855) & 7 & $0.50 \pm 1.61$ & 14.29 & 0 & & \\
\hline \multirow[t]{5}{*}{ TENEBRIONIDAE } & Phediodes sp. & 2 & $0.14 \pm 0.36$ & 14.29 & 0 & & \\
\hline & Prohylithus sp. & 41 & $2.93 \pm 8.01$ & 35.71 & 0 & & \\
\hline & Pilobalia sp. & 2 & $0.14 \pm 0.36$ & 14.29 & 0 & & \\
\hline & Psectrascelis subplanata Peña, 1994 & 4 & $0.29 \pm 0.61$ & 21.43 & 0 & & \\
\hline & Praocis obesa Kulzer, 1958 & 3 & $0.21 \pm 0.43$ & 21.43 & 1 & $0.07 \pm 0.27$ & 7.14 \\
\hline TROGIDAE & Polynoncus peruanus (Erichson, 1847) & 85 & $6.07 \pm 12.90$ & 42.86 & 0 & & \\
\hline
\end{tabular}



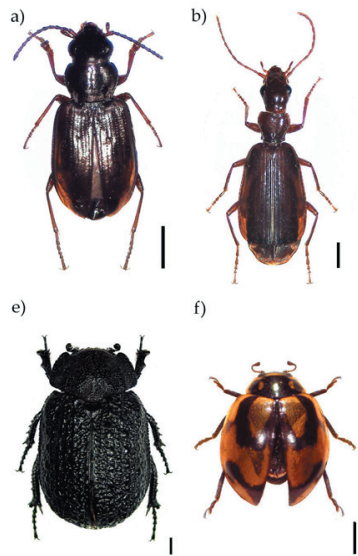

f)

g)
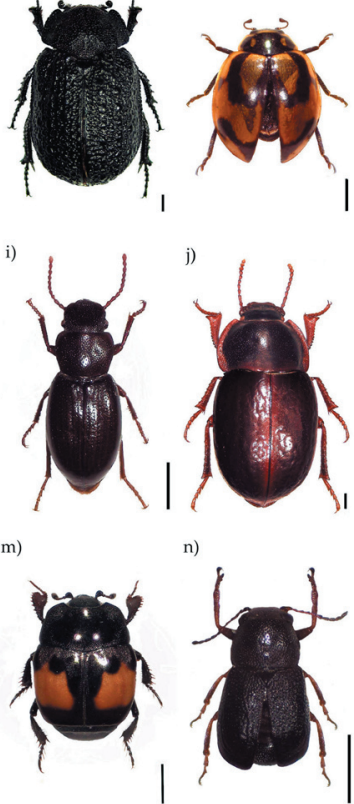
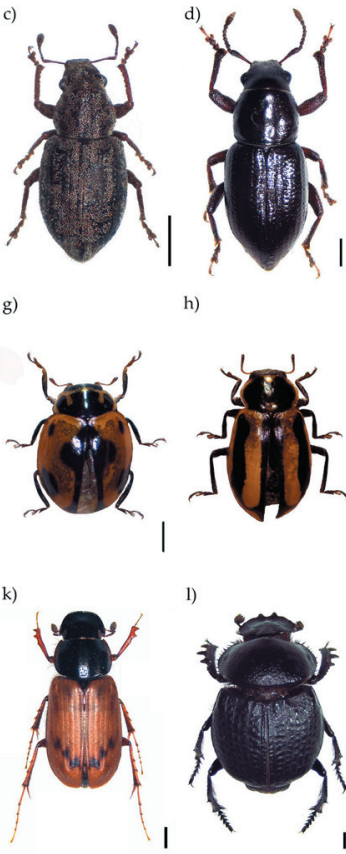

h)
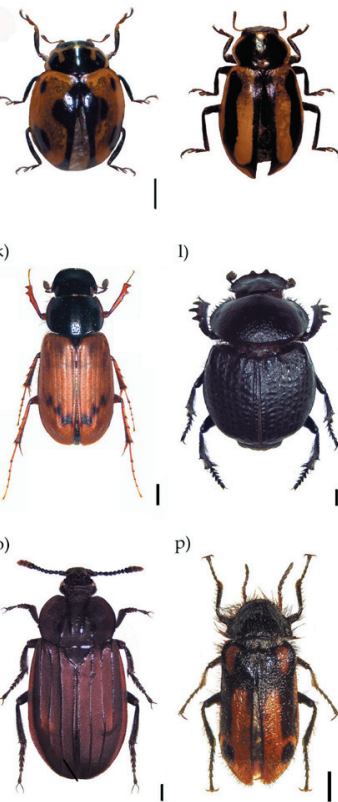

Figura 1. Coleoptera del bosque de Polylepis tomentella: a) Bembidion sp. 1, b) Lebiini sp. (Carabidae), c) Asymmathetes sp., d) Amitrus jelskei (Curculionidae), e) Polynoncus peruanus (Trogidae), f) Cycloneda arcula, g) Cycloneda sp., h) Eriopis nobilis (Coccinellidae), i) Prohylithus sp., j) Praocis obesa (Tenebrionidae), k) Podotenus sp., 1) Scybalophagus rugosus (Scarabaeidae), m) Euspilotus cf. decoratus (Histeridae), n) Pachybrachis sp. (Chrysomelidae), o) Oxelytrum anticola (Silphidae), p) Astylus subannulatus (Melyridae). Barra de escala: $1 \mathrm{~mm}$.

Figure 1. Coleoptera of the Polylepis tomentella woodlands: a) Bembidion sp. 1, b) Lebiini sp. (Carabidae),

c) Asymmathetes sp., d) Amitrus jelskei (Curculionidae), e) Polynoncus peruanus (Trogidae), f) Cycloneda arcula, g) Cycloneda sp., h) Eriopis nobilis (Coccinellidae), i) Prohylithus sp., j) Praocis obesa (Tenebrionidae), k) Podotenus sp., 1) Scybalophagus rugosus (Scarabaeidae), m) Euspilotus cf. decoratus (Histeridae), n) Pachybrachis sp. (Chrysomelidae), o) Oxelytrum anticola (Silphidae), p) Astylus subannulatus (Melyridae). Scale bar: $1 \mathrm{~mm}$.

casos en los que las diferencias morfológicas externas fueron muy sutiles o variables (como la microescultura elitral en Bembidion o el patrón de coloración en Astylus y Eriopis), se utilizó la morfología de la genitalia como carácter comparativo.

En la época húmeda, la riqueza registrada fue 42 especies, distribuidas en 17 familias. Curculionidae fue la familia más representativa $(S=9 ; 21.43 \%)$, seguida por Chrysomelidae y Tenebrionidae $(S=5$; $11.36 \%$ ). Las demás familias sólo estuvieron representadas por una o cuatro especies (Tabla 1). La frecuencia relativa osciló entre $7.14 \%$ (13 especies) y 57.14\% (1 especie). En la época seca se identificaron 12 especies, distribuidas en ocho familias. De éstas, Coccinellidae $(S=4$; $36.36 \%$ ) y Carabidae ( $\mathrm{S}=2 ; 18.18 \%$ ) fueron las más representativas. Las demás familias estuvieron representadas sólo por una especie (Tabla 1). La frecuencia relativa osciló entre $7.14 \%$ (siete especies) y $28.57 \%$ (dos especies). La diferencia de la riqueza entre ambas épocas estaría influenciada por la presencia de la flora asociada (Mendoza and Roque 2007) al parche de $P$. tomentella, que con la llegada de las lluvias encontraría suelos ricos en nutrientes y condiciones favorables para su desarrollo (Zamora 1996; Fjeldså 2002). Este resultado difiere de lo observado por Barrera (2001) en el páramo de Mucubají (Venezuela), donde el exceso de humedad podría no favorecer a la abundancia de escarabajos dado que disminuye notablemente su actividad. Además, en la época húmeda se reportaron especies que aparecieron con mayor frecuencia en las estaciones de evaluación. Esto podría suponer que se encontrarían mucho mejor distribuidas en el área que comprende al parche del bosque durante esa época, como lo observaron Amat et al. (1997) en un relicto del bosque altoandino de la cordillera oriental de Colombia.

Las familias Curculionidae, Carabidae, Chrysomelidae, Tenebrionidae y Coccinellidae fueron las mejor representadas. La estructura comunitaria de los coleópteros en cada época de muestreo estuvo compuesta por diferentes especies que responden a la disponibilidad de determinados recursos en el área del bosque de P. tomentella (Tabla 1 y Figura 1). La mayor permanencia de representantes de la familia Coccinellidae puede estar relacionada con que algunas de ellas parecen estar muy bien adaptadas a las zonas altoandinas del Perú (Bustamante et al. 2007).

Los estimadores no paramétricos ACE, ICE y Chao 2 sobreestimaron la riqueza observada con las trampas pasivas (Figura 2). Sin embargo, cuando se cruzaron estos datos con la riqueza total observada en el área de estudio (información de trampas Malaise y colecta activa), la estimación estuvo muy cerca de la riqueza observada (Chao2 $=43, \mathrm{ACE}=46$, ICE=47); ACE fue el mejor de los tres. Es posible que con un mayor esfuerzo de muestreo se 


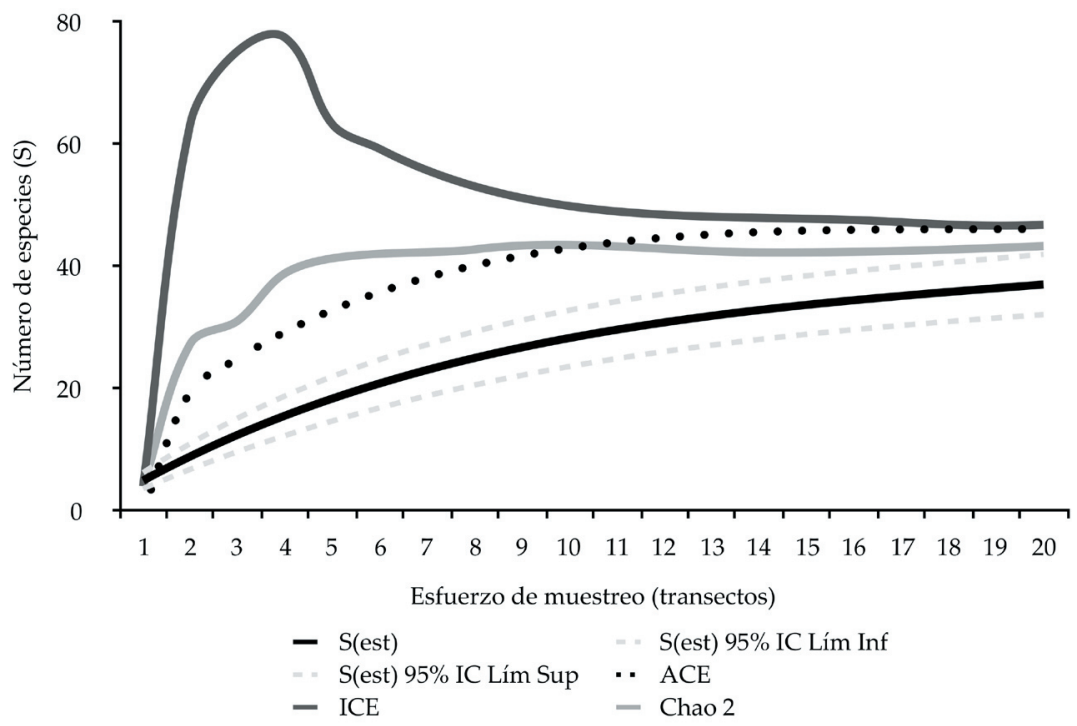

Figura 2. Curvas de acumulación de especies con estimadores no paramétricos. Cada transecto consistió en trampas de caída (7), trampas con cebo (3) y trampas cromotrópicas amarillas (2). Época húmeda: 1-10; época seca: 11-20; S(est): curva de riqueza estimada; IC Lím Inf 95\%: límite inferior del intervalo de confianza al 95\%; IC Lím Sup 95\%: límite superior del intervalo de confianza al 95\%.

Figure 2. Species accumulation curves with non-parametric estimators. Each transect had pitfall traps (7), bait traps (3) and chromotropic traps (2). Wet season: 1-10; dry season: 11-20; S(est): estimated richness curve; IC Lím inf 95\%: confidence interval lower bound at 95\%; IC Lím sup 95\%: confidence interval upper bound at 95\%.

puedan adicionar algunas otras especies que se considerarían raras (Gotelli and Colwell 2011), además de tener en cuenta la época de evaluación. La información obtenida puede servir para posteriores análisis de diversidad (complementándola como mínimo con un estudio de condiciones similares) y evaluar la variación de esos índices en el tiempo. Se sugiere estudiar la variación espacial y temporal de la riqueza y la diversidad de insectos en este tipo de hábitat, a fin de obtener información sobre el comportamiento de otras comunidades y su relación en estos ambientes. Los datos obtenidos se podrían usar para estimar el grado de perturbación de estos bosques relictos.

Agradecimientos. Nuestro agradecimiento al Consejo Superior de Investigación (CSI) de la Universidad Nacional Mayor de San Marcos por el financiamiento al proyecto 101001291. Un reconocimiento especial a R. Westerduijn, G. del Río, J. Morrone, G. Flores, A. Giraldo, P. Perkins, G. González, A. Smith, F. Aballay y R. Constantin por su colaboración en la identificación de los especímenes. A M. Huamán y C. Carranza por su ayuda en la labor de campo y al señor R. Ayvar por su importante apoyo logístico.

\section{REFERENCIAS}

Amat, G., A. Lopera, and S. J. Amézquita. 1997. Patrones de distribución de escarabajos coprófagos (Coleoptera: Scarabaeidae) en relicto del bosque altoandino, cordillera oriental de Colombia. Caldasia 19:191-204.

Ashe, J. S., and R. A. B. Leschen. 1995. Cajachara carltoni, a new genus and species of rove beetle (Coleoptera Staphylinidae Aleocharinae) from an Ecuadorean paramo. Tropical Zoology 8(1):85-93. DOI: 10.1080/03946975.1995.10539274.

Asociación Ecosistemas Andinos (ECOAN). 2005. Evaluación de la biodiversidad de los bosques de Polylepis del corredor de Conchucos-Huaráz. Informe presentado a Conservación Internacional.

Asociación Ecosistemas Andinos (ECOAN). 2006. Evaluación de la biodiversidad de los bosques de Polylepis de la Región Puno. Informe presentado a American Bird Conservancy y Gordon and Betty Moore Foundation.

Asociación Ecosistemas Andinos (ECOAN). 2007. Evaluación de la biodiversidad de los Bosques de Polylepis en la zona sur oeste del Parque Nacional Otishi. Informe presentado a Conservación Internacional - Perú.

Barrera, M. R. 2001. Análisis comparativo de la comunidad de coleópteros epígeos en dos bosques de Polylepis sericea. Páramo de Mucubají, Mérida-Venezuela. Tesis de Licenciatura en Biología. Universidad de Los Andes, Mérida. Venezuela. Ecotropicos 14(1)

Bustamante, A., G. González, and A. Oróz. 2007. Revisión y descripción de algunas especies de Eriopis (Coleoptera: Coccinellidae) del Perú. Boletín Sociedad Entomológica Aragonesa 41:67-72.

Bustamante, A. A., E. Yábar, E. L. Marquina, and A. Elme. 2017. Primer registro del género Stenadalia Weise 1926

(Coleoptera: Coccinelidae) en el Perú. Arquivos Entomolóxicos 17:459-462.

Centro de Biodiversidad y Genética (CBG). 2008. Estudio de la biodiversidad de la subcuenta del río "Jatun Mayu". 
Facultad de Ciencias y Tecnología, Universidad Mayor de San Simón, Bolivia. Working paper Nº4-08.

Chaboo, C. 2015. Beetles (Coleoptera) of Peru: A survey of the families. Part I. Overview. Journal of the Kansas Entomological Society. 88(2):135-139.

Colwell, R. K. 2013. EstimateS: Statistical estimation of species richness and shared species from samples. Version 9. User's Guide and application published at: purl.oclc.org/estimates.

Didham, R. K., P. M. Hammond, J. H. Lawton, P. Eggleton, and N. E. Stork. 1998. Beetle species responses to tropical forest fragmentation. Ecological Monographs 68:295-323.

Ellenberg, H. 1958. Wald oder Steppe? Die naturliche Pflanzendecke der Anden Perus. Umschau in Wis-senschaft und Technik 21:645-681.

Fjeldså, J. 2002. Polylepis forests - vestigies of a vanishing ecosystem in the Andes. Ecotropica 8:111-123.

Fuentes, P. V., and E. Camero. 2006. Estudio de la fauna de escarabajos coprófagos (Coleoptera: Scarabaeidae) en un bosque húmedo tropical de Colombia. Entomotropica 21:13-143.

Gonzales, J. A., A. A. De la Fuente Díaz, L. Hernández, D. Buzo, and C. Bonache. 2010. Evaluación de estimadores no paramétricos de la riqueza de especies. Un ejemplo con aves en áreas verdes de la ciudad de Puebla, México. Animal Biodiversity and Conservation 33:31-45.

Gotelli, N. J., and R. K. Colwell. 2011. Estimating species richness. Pp. $39-54$ in A. E. Magurran and B. J. McGill (eds.). Biological diversity frontiers in measurement and assessment. Oxford University Press Inc., New York, USA.

Gullan, P., and P. Cranston. 2010. The insects: an outline of entomology. Chapman and Hall. London. UK.

Hamel, A. C., S. K. Herzog, D. J. Mann, T. H. Larsen, B. D. Gill, W. D. Edmonds, and S. Spector. 2009. Distribución e historia natural de escarabajos coprófagos de la tribu Phanaeini (Coleoptera: Scarabaeidae: Scarabaeinae) en Bolivia. Kempffiana 5(2):43-95.

Kessler, M. 2002. The "Polylepis problem": where do we stand. Ecotropica 8:97-110.

Lawrence, J. F., and A. F. Newton, Jr. 1995. Families and subfamilies of Coleoptera (with selected genera, notes, references and data on family-group names). Pp. 779-1006 in J. Pakaluk and S. A. Slipinski (eds.). Biology, Phylogeny, and Classification of Coleoptera: Papers Celebrating the 80th Birthday of Roy A. Crowson. Museum i Instytut Zoologii PAN, Warszawa.

Lloyd, H. 2008. Foraging ecology of high andean insectivorous birds in remnant Polylepis forest patches. The Wilson Journal of Ornitology 129(3):531-544.

López, A. M., and G. Williams. 2006. Evaluación de métodos no paramétricos para la estimación de riqueza de especies de plantas leñosas en cafetales. Boletín de la Sociedad Botánica de México 78:7-15.

Lozada, A., I. Fernández, and M. Trujillo. 2004. Lista preliminar de los Coleópteros (Insecta, Coleoptera) de Topes de Collantes, Trinidad, Sancti Spiritus, Cuba. Boletín Sociedad Entomológica Aragonesa 34:101-106.

Méndez, D. M., M. M. López, and R. García. 2012. Diversidad de escarabajos (Coleopteros, Staphylinidae) en bosques altoandinos restaurados de los Andes centrales de Colombia. Revista Colombiana de Entomología 38(1):141-147.

Mendoza, W., and A. Cano. 2011. Diversidad del género Polylepis (Rosaceae, Sanguisorbeae) en los Andes peruanos. Revista Peruana de Biología 18:197-200.

Mendoza, W., and J. E. Roque. 2007. Diversidad de la flora vascular asociada a los bosques de Polylepis (Rosaceae) en los andes meridionales del Perú (Ayacucho): implicancias para su conservación. Serie de publicaciones de Flora y Fauna Silvestre. Instituto Nacional de Recursos Naturales, Lima, Perú.

Meneses L. A., and J. A. Camacho. 2016. Dieta de aves en bosques de Polylepis quadrijuga (Rosaceae) en el páramo de la Rusia, Duitama (Boyacá - Colombia). Revista Ciencias Agropecuarias 2:22-27.

Mora, E. 2015. Efecto de cambio climático sobre los modelos de distribución de la abundancia en ensamblajes animales en la isla de Tenerife: caso de los coleópteros de la superficie del suelo (Carabidae) como indicadores ecológicos. Tesis de grado. Universidad de Laguna. España.

Nichols, E., T. Larsen, S. Spector, A. L. Davis, F. Escobar, M. Favila, K. Vulinec, and The Scarabaeinae Research Network. 2007. Global dung beetle response to tropical forest modification and fragmentation: a quantitative literature review and meta-analysis. Biological Conservation 137:1-19.

Otavo, S. E., A. P. Roselli, and J. A. Noriega. 2013. Superfamilia Scarabaeoidea (Insecta: Coleoptera) como elemento bioindicador de perturbación antropogénica en un parque nacional amazónico. Revista de Biología Tropical 61: 735-752.

Qodri, A., R. Raffiudin, and W. A. Noerdjito. 2016. Diversity and abundance of Carabidae and Staphylindae (Insecta: Coleoptera) in four montane habitat types on Mt. Bawakaraeng, South Sulawesi. Journal of Biosciences 23:22-28.

Perrault, G. G. 1994. Ecobiogeography of Carabidae in the Andes of Venezuela. Pp. 45-49 in K. Desender et al. (eds.). Carabid Beetles: Ecology and Evolution. Kluwer Academic Publishers.

Stotz, D. F., J. W. Fitzpatrick, T. A. Parker, and D. K. Moskovits. 1996. Neotropical birds: ecology and conservation. University of Chicago Press, Chicago, Illinois, USA.

Triplehorn, Ch., N. Johnson, and D. Borror. 2005. Borror and DeLong's introduction to the study of insects. Thompson Brooks/Cole, Belmont, CA, USA.

Velez, V., J. Cavelier, and B. Devia. 1998. Ecological traits of the tropical treelike species Polylepis quadrijuga (Rosaceae) in the Andes of Colombia. Journal of Tropical Ecology 15:771-781.

Zamora, C. 1996. Las regiones ecológicas del Perú. Pp. 137-142 en L. Rodríguez (ed.). Diversidad Biológica del Perú. Proyecto FANPE, GTZ-INRENA. Lima, Perú.

Zutta, B., P. Rundel, S. Saatchi, J. Casana, P. Gauthier, A. Soto, Y. Velazco, and W. Buermann. 2012. Prediciendo la distribución de Polylepis: bosques Andinos vulnerables y cada vez más importantes. Revista Peruana de Biología 19:205-212. 